\title{
Guidelines for Submission of Spontaneous Regression of Childhood Gliomas
}

All cases of spontaneous involution or regression of childhood low-grade gliomas are to be sent to the Editorial Office of Pediatric Neurosurgery. The following information is requested:

(1) A clinical summary of the patient, including age at diagnosis, clinical presentation, date of surgery, a copy of the operative report, and date of follow-up examination showing regression or involution and follow-up data. Specific comments on associated medical or neurological conditions, including whether the child had neurofibromatosis.

(2) Copies of appropriate neuroradiographic studies performed preoperatively, postopera-tively and at time of regression or involution.

\section{0 \\ Gajjar/Heideman/Kovnar/Langston/ Sanford/Douglass/Jenkins/Horowitz/Kun \\ Chemotherapy in Low Grade Gliomas}

with newly diagnosed disease and those children who have significant residual disease after attempts at surgical resection. Are the goals of treatment to delay the need for radiotherapy or to obviate the need for radiotherapy? At what age is it safe to deliver local radiotherapy and what side effects are acceptable if the goals of treatment are to delay the need for radiotherapy? Is is reasonable to utilize potentially neurotoxic, expecially ototoxic, chemotherapy in patients with lesions which have already impaired vision? It is hoped that the next generation of chemother-apeutic trials in children with low-grade gliomas will pro-spectively address these issues.

Critical to the evaluation of any form of therapy for pediatric low-grade gliomas is an understanding of the natural history of these tumors, including whether surgical intervention changes the biology of the lesions. There have been anecdotal reports of spontaneous or surgically induced involution and/or regression of lesions after less than total surgical resection, but the frequency of this occurrence is unknown [3]. Obviously, if this tendency is a frequent one, postsurgical reports of radiotherapy- or chemotherapy-induced tumor shrinkage take on less significance. In an attempt to address the issue of spontaneous involution, the editors of Pediatric Neurosurgery will set up a registry for cases of spontaneous involution. It

is hoped that pediatric neurosurgeons around the world will send their cases of spontaneous regression (either without surgical intervention or after surgical intervention) to the journal for critical review and compilation. Without this registry, it is unlikely that this event can ever be appropriately characterized.

References 1 Packer RJ, Sutton LN, Bilanuik LT, et al:

Treatment of chiasmatic/hypothalamic gliomas of childhood with chemotherapy, an updated report. Ann Neurol 1988;23:79-85.

2 Wisoff JH, Abbott IR, Epstein F: Surgical management of exophytic chiasmatic-hypotha-lamic tumors of childhood. J Neurosurg 1990; 73:661-667.

Rutka JT, Hoffman HJ, Drake JM, Humphreys RP: Suprasellar and sellar tumors in childhood and adolescence. Neurosurg Clin NA 1992;3:803-820.

Epstein F, McCleary EL: Intrinsic brainstem tumors of childhood: Surgical indications. J Neurosurg 1986;64:1 1-15.

5 Packer RJ, Finlay J, Lange B, et al: Efficacy of chemotherapy (CTx) with carboplatin (CP) and vincristine (VCF) for children with low grade gliomas (LGGs). Ann Neurol 1992;32: 458. 